\title{
UTILIZAÇ̃̃O DO GPR PARA CARACTERIZAR TAMBORES PLÁSTICOS ENTERRADOS NO SÍTIO CONTROLADO DE GEOFÍSICA RASA DO IAG/USP
}

\author{
Selma Isabel Rodrigues ${ }^{1}$ e Jorge Luís Porsani \\ Recebido em 7 dezembro, 2005 / Aceito em 19 abril, 2006 \\ Received on December 7, 2005 / Accepted on April 19, 2006
}

\begin{abstract}
In this work, the results obtained through the use of GPR - Ground Penetrating Radar method in characterization of plastic drums buried within the Shallow Geophysical Controlled Test Site of the IAG, installed inside of São Paulo University/USP are presented. The plastic drums are used to simulate of environmental contamination in urban areas. Empty drums and drums filled with fresh and salty water, are used to simulate respectively resistive contaminant, water from nuclear reactors, and conductive contaminant. Measurements were carried out before the installation of the targets in subsurface, in order to obtain data from "background" of the land, or geologic horizons before disturbances. Also bidimensional synthetic GPR models were constructed to simulate the geophysical response, to help in data interpretation and to decrease the uncertainties in the results. In a second stage, GPR data were obtained after targets installation. The analysis of the results was carried through the integration of real GPR data, 2D GPR numerical modeling, and the known depths of targets. The data integration presented an excellent agreement, allowing the clarity identification of all the targets, and their related geophysical signature. The qualitative and quantitative results on IAG's Test Site can be used in places where there is no have any information about the subsurface.
\end{abstract}

Keywords: GPR-Ground Penetrating Radar, Shallow Geophysical Controlled Test Site-IAG/USP, Plastic drums, Numerical modeling GPR 2D, Environmental contamination.

RESUMO. Neste trabalho são apresentados os resultados obtidos a partir da utilização do método GPR - Ground Penetrating Radar na caracterização de tambores plásticos enterrados no Sítio Controlado de Geofísica Rasa do IAG instalados dentro do campus da Universidade de São Paulo - USP. Os tambores plásticos simulam estudos de contaminação ambiental em áreas urbanas. Os tambores enterrados vazios, com água doce e com água salgada, representam respectivamente contaminantes resistivos, água proveniente de reatores nucleares e contaminantes condutivos. Na primeira etapa, os trabalhos de campo foram realizados antes da disposição dos alvos em subsuperfície, visando obter dados referentes ao "background" do terreno, ou seja, dos horizontes geológicos anteriores às perturbações. Foram construídos modelos sintéticos bidimensionais para simular numericamente as respostas geofísicas, visando auxiliar na interpretação dos dados e diminuir as incertezas nos resultados. Na segunda etapa, foram obtidos dados de GPR após a instalação dos alvos. A análise dos resultados foi realizada através da integração dos dados reais de GPR, das modelagens numéricas GPR 2D e das profundidades conhecidas dos alvos, os quais apresentaram uma excelente concordância, permitindo identificar com clareza todos os alvos e distingui-los quanto à assinatura geofísica. Os resultados qualitativos e quantitativos sobre o Sitio Controlado do IAG poderão ser utilizados em locais onde há pouca ou nenhuma informação sobre a subsuperfície.

Palavras-chave: GPR-Ground Penetrating Radar, Sítio Controlado de Geofísica Rasa-SCGR-IAG/USP, Tambores plásticos, Modelagem numérica GPR 2D, contaminação ambiental.

\footnotetext{
1Pós-Graduação em Geofísica - IAG-USP. Rua do Matão, 1226 - Cidade Universitária, 05508-090 São Paulo, SP, Brasil. Tel: (11) 3091-2791; Fax: (11) 3091-5034 -E-mail: selma@iag.usp.br

${ }^{2}$ IAG-USP - Departamento de Geofísica, Rua do Matão, 1226 - Cidade Universitária, 05508-090 São Paulo, SP, Brasil. Tel: (11) 3091-4734; Fax: (11) 3091-5034 -E-mail: porsani@iag.usp.br
} 


\section{INTRODUÇÃo}

0 aumento da demanda populacional nos grandes centros urbanos e a utilização descontrolada dos recursos naturais acarreta impactos ambientais os quais limitam a qualidade de vida e 0 desenvolvimento social. Este contexto tem contribuído para o aumento de trabalhos relacionados a estudos ambientais, planejamento urbano e geotecnia, visando remediar e recuperar áreas contaminadas além da adoção de políticas de gerenciamentos para 0 uso do solo e subsolo que se adaptem à realidade social.

Atualmente, as investigações geofísicas não destrutivas apresentam-se como ferramentas significativas na caracterização dos problemas ambientais e têm ampla aceitação na sociedade devido aos baixos custos e à otimização na execução dos levantamentos de campo. Conforme a literatura específica, os métodos geoelétricos são os mais eficazes e adequados para a identificação de alvos enterrados e mapeamento de plumas de contaminação (Davis \& Annan, 1989; Daniels et al., 1994; Reynolds, 1997; Atekwana et al., 2000; Porsani et al., 2004a).

Os estudos ambientais sob condições controladas com a utilização de tambores metálicos e plásticos vêm aumentando significativamente nos últimos anos. Bowders Jr. et. al. (1982) trabalharam com a caracterização destes alvos utilizando o Método GPR-Ground Penetrating Radar e Lord Jr. \& Koerner (1988) utilizaram a integração de métodos geofísicos: Indução Eletromagnética, Detecção de Metal, Magnetometria e GPR. A aplicação da geofísica na engenharia civil também é notória, por exemplo, Olhoeft et al. (1994) desenvolveram estudos sobre o GPR utilizando diversos alvos em subsuperfície, tais como, tubulações, fios elétricos, minas metálicas e plásticas; Zeng \& McMechan (1997) simularam numericamente respostas com o Método GPR para diversas tubulações e tanques enterrados na subsuperfície; e Grandjean et al. (2000) testaram diferentes técnicas do GPR aplicadas à caracterização de alvos encontrados no contexto urbano, tais como, blocos de concreto, vazamento de água, tubulações de ferro e PVC preenchidas com água ou vazias, cavernas etc. Algumas universidades também vêm desenvolvendo projetos de pesquisa junto a áreas de testes controlados para avaliar a eficiência dos métodos geofísicos (Sauck, 1996; Szeto, 1996; Mabee \& Brown, 2003; Porsani et al., 2004b, 2006).

Entretanto, a aplicação dos métodos geofísicos em áreas urbanas ainda representa um desafio para os geocientistas em razão da intensa ocupação da subsuperfície (tubulações de água, gás, luz, esgotos, cabos elétricos, cabos de fibra ótica; estacionamentos etc), pavimentação, ruídos e à complexidade no processo de interpretação dos resultados. Para entender e diminuir as impre- cisões encontradas na interpretação geofísica, o Sítio Controlado de Geofísica Rasa - SCGR do Instituto de Astronomia, Geofísica e Ciências Atmosféricas - IAG foi instalado dentro do campus da Universidade de São Paulo - USP (PORSANI et al., 2006).

A área de estudos no SCGR está situada sobre a borda da Bacia Sedimentar de São Paulo (Figura 1), sendo caracterizada por um aterro com aproximadamente $3 \mathrm{~m}$ de espessura, composto por um material argilo-arenoso de cor vermelha escura (Borges, 2002). Entre a base do aterro e o embasamento granito-gnáissico há a predominância de $53 \mathrm{~m}$ de sedimentos areno-argilosos das formações Resende e São Paulo (Porsani et al., 2004b).

A área de testes controlados (SCGR-IAG) abrange sete linhas que são caracterizadas por diferentes materiais dispostos até $2 \mathrm{~m}$ de profundidade, e que simulam problemas geotécnicos, ambientais e arqueológicos (Porsani et al., 2006). 0 estudo do SCGR permite que as informações geofísicas obtidas através da calibração dos alvos possam ser utilizadas em áreas onde não se têm informações da subsuperfície. Alguns resultados sobre as investigações geofísicas dentro da área do SCGR podem ser encontrados em Porsani et al. (2004b, 2006), Borges et al. (2004), Rodrigues (2004) e Silva (2004).

Neste trabalho são apresentados os resultados obtidos através da caracterização geofísica de tambores plásticos dispostos sobre uma das sete linhas do SCGR utilizando o método GPR - Ground Penetrating Radar, mostrando a importância da geofísica aplicada a estudos ambientais controlados. Os tambores plásticos foram enterrados vazios, preenchidos com água doce e com água salgada, visando simular contaminantes resistivos e condutivos na subsuperfície.

Para dar mais confiabilidade na interpretação dos dados e reduzir as incertezas em sua interpretação, modelagens numéricas bidimensionais foram realizadas utilizando-se 0 método das Diferenças Finitas no Domínio do Tempo - FDTD (Yee, 1966; Ward \& Hohmann, 1987). A comparação entre os resultados das modelagens 2D, com as seções de GPR e as profundidades conhecidas dos alvos permitiu obter assinaturas geofísicas diferenciadas referentes ao tipo de material que constitui 0 tambor e 0 seu conteúdo.

\section{MÉTODOS}

\section{Ground Penetrating Radar}

0 GPR - Ground Penetrating Radar é um método geofísico que se fundamenta na propagação e reflexão de ondas eletromagnéticas em altas freqüências (1 MHz a 2,5 GHz). Os traços de GPR são obtidos através da movimentação das antenas transmis- 


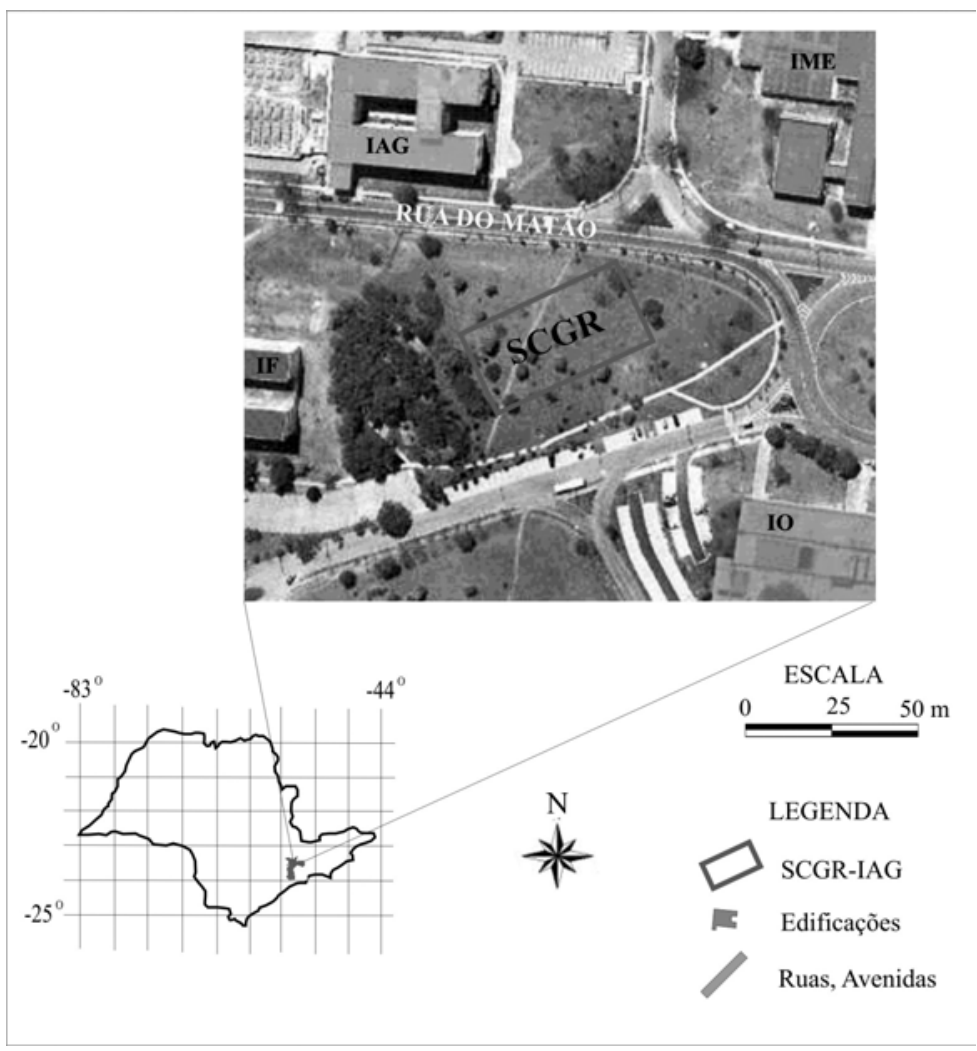

Figura 1 - Localização da área de estudos, São Paulo.

sora e receptora ao longo de um perfil. A antena transmissora irradia pulsos eletromagnéticos para a subsuperfície e a receptora registra os sinais refletidos na interface entre os alvos que apresentam propriedades eletromagnéticas contrastantes (permissividade dielétrica, condutividade elétrica e permeabilidade magnética). Esta energia refletida permite deduzir informações sobre alvos pontuais, estruturas inclinadas ou horizontais, de acordo com 0 formato do refletor. A Figura 2 mostra o percurso esquemático da onda eletromagnética refletida num alvo pontual. Os sinais eletromagnéticos são registrados através de sistemas digitais de acordo com o tempo de percurso entre a irradiação e a reflexão dos campos eletromagnéticos, medidos em nano-segundos (ns). Os princípios físicos e matemáticos envolvidos na propagação de ondas eletromagnéticas em qualquer meio estão fundamentados nas equações de Maxwell (Reitz et al., 1982). Informações detalhadas sobre o procedimento desta metodologia podem ser encontradas na literatura (Davis \& Annan, 1989; Daniels, 1996; Porsani, 1999), dentre outras.

Para se obter a localização dos alvos em profundidade faz-se necessário conhecer a velocidade de propagação que a onda eletromagnética viaja na subsuperfície. A velocidade pode ser obtida de três formas: utilizando a expressão $(v=2 h / t)$ na qual relaciona os valores reais de profundidades das interfaces e 0 tempo de percurso das reflexões medido no radargrama; ajustando-se as curvas de velocidades aos refletores, através do método $x^{2}-t^{2}$, uma aproximação da análise da Equação de Dix para determinar as velocidades da camadas e as espessuras (Burger, 1992); ou através de levantamentos de campo utilizando as técnicas de sondagens de velocidades CMP - Common Mid Point e WARR Wide Angle Reflection and Refraction. A conversão do tempo em profundidade dos dados de GPR, apresentados neste trabalho, foi realizada através do ajuste das curvas de velocidades nos refletores. A profundidade de investigação é inversamente proporcional à frequiência, ou seja, quanto maior a freqüência da antena menor será a profundidade de investigação; e também sofre limitação quando 0 ambiente apresenta materiais altamente condutivos.

\section{Modelagem numérica bidimensional}

Os estudos sintéticos referentes às modelagens numéricas bidimensionais são passos importantes na aplicação dos métodos geofísicos em situações reais de campo. A modelagem numérica 


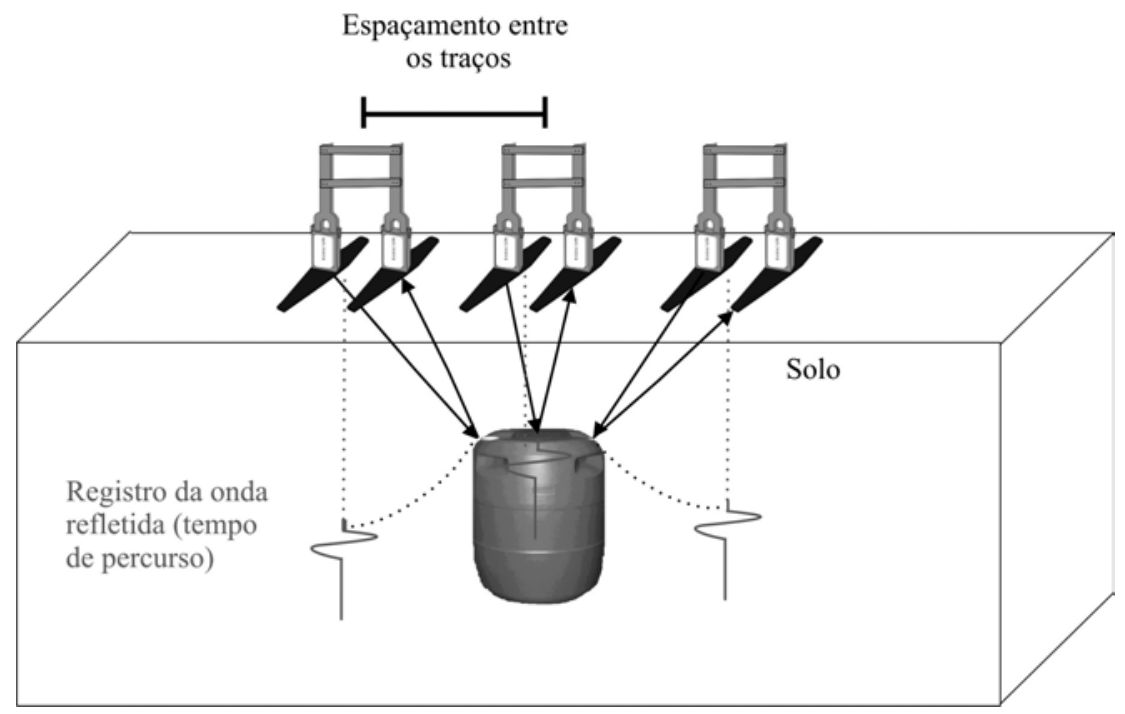

Figura 2 - Esquema da reflexão da onda eletromagnética num tambor plástico.

auxilia na escolha dos melhores parâmetros para a aquisição dos dados e verifica se as respostas geofísicas serão significativas para o trabalho. Além disso, proporciona maior confiabilidade na interpretação dos dados reais, diminuindo incertezas que venham a comprometer a credibilidade dos resultados. Para estudar o comportamento da propagação e reflexão de campos eletromagnéticos foi utilizado o método numérico das Diferenças Finitas no Domínio do Tempo - FDTD (Yee, 1966; Ward \& Hohmann, 1987; Taflove \& Hagness, 2000).

0 método FDTD baseia-se na implementação de códigos matemáticos computacionais através de aproximações numéricas sucessivas, substituindo as equações diferenciais por diferenças finitas. Esta substituição ocorre pela expansão das equações diferenciais em Série de Taylor e truncamento ao nível da ordem de erro desejada, ou seja, segunda ordem. Desta forma, as equações de Maxwell são resolvidas por equações algébricas, através do conceito de discretização desenvolvido por Euler, na qual as taxas relacionadas às derivadas são substituídas por incrementos espaciais $(\Delta x)$ e temporais $(\Delta t)$. 0 modelo é discretizado em um grid composto por um conjunto finito de pontos, representados pelos chamados nós da malha, cada nó representa um vetor de campo com propriedades elétricas e magnéticas (Belém, 2001; Costa, 2002).

Para gerar as simulações foi utilizado o software Reflexw (Sandmeier, 2004), e as freqüências centrais de 100, 200 e 500 $\mathrm{MHz}$. Os valores de $\Delta t$ são calculados a partir dos valores de $\Delta x$, respeitando 0 critério de estabilidade numérica (Belém, 2001). As condições de contorno absorventes é uma escolha im- portante para o método por limitar no espaço a região infinita, proporcionando uma diminuição de reflexões espúrias dos contornos do modelo, através do aumento gradativo da condutividade, alcançando um valor máximo nas extremidades. 0 campo de ondas foi gerado utilizando a fonte exploding reflector, ou seja, as ondas são geradas simultaneamente a partir do alvo refletor e enviadas para a superficie, simulando uma seção de GPR zero-offset (Yilmaz, 1987). Quanto à polarização das antenas foi utilizado 0 modo TE (Transversal Elétrico), ou seja, as antenas transmissora e receptora foram simuladas e posicionadas paralelamente e ortogonalmente à direção do perfil, visando maximizar a resposta das reflexões em subsuperfície (Annan, 1992; Versteeg, 1996).

\section{AQUISIÇÃO E PROCESSAMENTO DAS SEÇÕES DE GPR}

As seções de GPR foram adquiridas com 0 equipamento Ramac. As aquisições foram realizadas em dois períodos: "antes" e "depois" da instalação dos alvos no SCGR do IAG, utilizando-se a técnica common offset (perfil de reflexão com afastamento constante), tanto no modo passo-a-passo como no modo contínuo. 0 primeiro período de aquisição dos dados teve por finalidade mapear os contrastes entre as propriedades físicas da litologia da área, ou seja, do background. E no segundo período, a caracterização dos alvos já dispostos nas profundidades préestabelecidas. Ambas etapas foram realizadas utilizando-se as antenas blindadas (100 e $500 \mathrm{MHz}$ ) e não blindadas (100 e $200 \mathrm{MHz}$ ). Sob a linha de estudo, com $30 \mathrm{~m}$ de comprimento, existem tambores plásticos de 100 litros enterrados até $2 \mathrm{~m}$ de profundidade. 0 espaçamento adotado entre os traços foi de 
0,05 m e stack (empilhamento dos traços) de 32 para o modo contínuo e de 0,2 me stack de 256 para o modo passo-a-passo. Em todos os perfis foram utilizadas 512 amostras por traço e 0 padrão de radiação das antenas no modo TE.

0 software Gradix foi empregado para processar os dados de GPR, proporcionando uma melhor visualização do perfil e a caracterização dos alvos de interesse. Para o refinamento destes dados foram usadas ferramentas básicas, dentre elas: correção do tempo zero, análise dos espectros de freqüências, filtragens temporais e espaciais, ganhos no tempo (lineares, esféricos e exponenciais), análise de velocidades e a conversão do tempo em profundidade.

\section{RESULTADOS E DISCUSSÕES}

Neste item são apresentados e discutidos os resultados obtidos através das simulações numéricas e as seções de GPR adquiridas "antes" e "depois" dos tambores plásticos terem sido instalados no SCGR-IAG.

\section{Modelagem numérica bidimensional}

Os estudos de modelagem numérica GPR 2D dos tambores plásticos instalados no SCGR-IAG foram realizados para se obter confiabilidade e reduzir as incertezas relativas à interpretação dos resultados. Os parâmetros utilizados na construção do modelo sintético são apresentados nas Tabelas $1 \mathrm{e} 2$, que indicam as dimensões dos alvos e as propriedades físicas dos materiais, respectivamente. Na Figura 3a é mostrado o modelo sintético construído para a Linha de tambores plásticos instalados no SCGRIAG. Os tambores plásticos foram instalados na vertical vazios (alvos 1 e 8), cheios de água doce (alvos 2, 7 e 9), semipreenchidos com água salgada (alvos 3 e 10) e cheio de água salgada (alvos 4,5 e 11). Nas Figuras 3b, 3c e 3d são apresentados os resultados das modelagens numéricas para as antenas de 100, 200 e $500 \mathrm{MHz}$, respectivamente. É importante ressaltar que além dos tambores, um cano metálico transversal às linhas instaladas na área de estudos também foi modelado. 0 cano metálico com 3,8 cm de diâmetro foi instalado para referenciar todas as linhas instaladas no SCGR, servindo como alvo-guia.

Conforme se observa nos radargramas sintéticos, todos os alvos pontuais foram caracterizados por reflexões hiperbólicas. Os tambores vazios (alvos 1 e 8) são caracterizados por fortes reflexões no topo. A base dos tambores não pode ser identificada, pois a onda eletromagnética dentro destes tambores viaja com a velocidade da luz, e 0 tempo de percurso entre 0 topo e a base menor que $5 \mathrm{~ns}$. Portanto, ocorre uma sobreposição do primeiro refletor (topo do tambor) e do segundo refletor (base do tambor).
Tabela 1 - Dimensão dos tambores plásticos e do cano metálico instalados no SCGR-IAG.

\begin{tabular}{|c|c|c|c|}
\hline Alvos & $\begin{array}{c}\text { Espessura do } \\
\text { material }(\mathrm{cm})\end{array}$ & $\begin{array}{c}\text { Comprimento } \\
(\mathrm{cm})\end{array}$ & $\begin{array}{c}\text { Diâmetro } \\
(\mathrm{cm})\end{array}$ \\
\hline $\begin{array}{c}\text { Tambores } \\
\text { plásticos }\end{array}$ & 0.175 & 69 & 47 \\
\hline $\begin{array}{c}\text { Cano } \\
\text { metálico }\end{array}$ & 0.13 & 5000 & 3.8 \\
\hline
\end{tabular}

Tabela 2 - Propriedades físicas dos materiais (Annan, 1992; Zeng \& McMechan, 1997, Grandjean et al., 2000).

\begin{tabular}{|c|c|c|c|}
\hline Material & $\varepsilon_{r}$ & $\mu_{r}$ & $\sigma(\mathrm{S} / \mathrm{m})$ \\
\hline Solo argiloso (background) & 18 & 1 & $10^{-2}$ \\
\hline Plástico & 3.3 & 1 & 0 \\
\hline Ar & 1 & 1 & 0 \\
\hline Água doce & 81 & 1 & $5 \times 10^{-4}$ \\
\hline Água salgada & 81 & 1 & 3 \\
\hline Metal & 300 & 100 & $10^{10}$ \\
\hline
\end{tabular}

Para os tambores preenchidos com água doce (alvos 2, $7 \mathrm{e}$ 9), dispostos a 1, 1,5 e 0,5 m de profundidade, respectivamente, observam-se duas reflexões em tempos diferentes (Figura 3b). 0 primeiro refletor caracteriza o topo e o segundo refletor está relacionado com a base do tambor. A explicação para estes refletores é obtida através do estudo dos tempos teóricos para a reflexão da onda eletromagnética tanto no topo quanto na base dos alvos (Tabela 3). Os refletores no topo dos tambores apresentam uma inversão de polaridade em relação às reflexões geradas no topo dos tambores vazios devido ao elevado contraste de impedância entre 0 solo argiloso e a água. A baixa velocidade de propagação da onda eletromagnética na água (0,033 m/ns) propicia um tempo maior de percurso entre o topo e a base destes tambores. Os refletores menos acentuados (topo dos tambores) devem-se a interface solo argiloso/plástico/topo da água doce, o qual provoca uma atenuação do sinal, devido à condutividade elétrica no solo argiloso (aproximadamente $10 \mathrm{mS} / \mathrm{m}$ ) ser maior que a da água doce $(0,5 \mathrm{mS} / \mathrm{m})$.

Os tambores semipreenchidos com água salgada (alvos 3 e 10) foram caracterizados por refletores gerados na interface solo/plástico/ar. 0 limite superior da água salgada não é identificado, devido à sobreposição dos refletores do topo do tambor (vazio) e o topo da água salgada semelhante aos tambores vazios (alvos $1 \mathrm{e}$ 8). A base destes tambores não é determinada devido à elevada condutividade elétrica da água salgada, provocando uma elevada atenuação do sinal. 


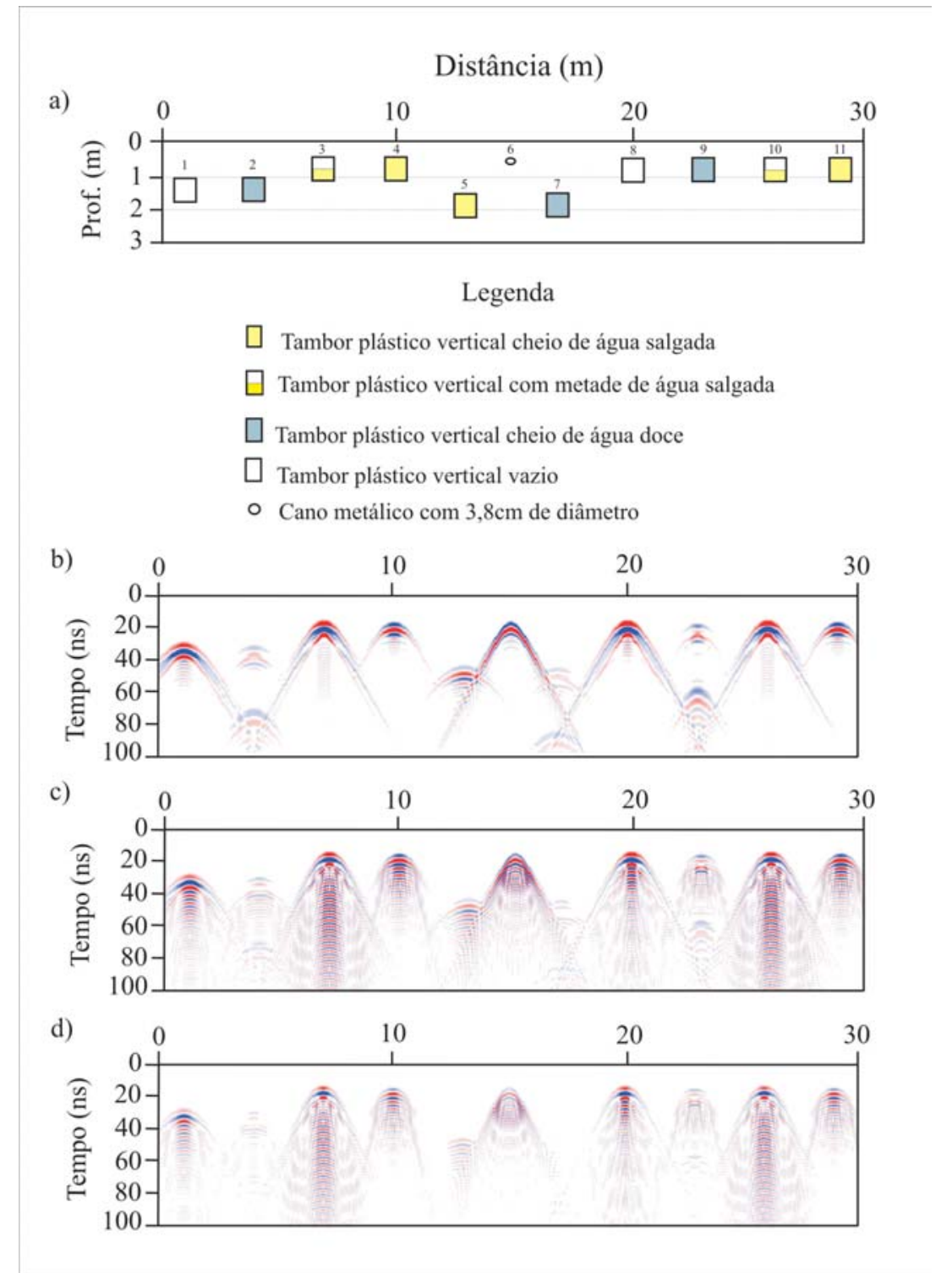

Figura 3 -a) Modelo sintético dos tambores plásticos na subsuperfície, b) Resultados das simulações numéricas com as freqüências de $100 \mathrm{MHz}$, c) $200 \mathrm{MHz}$ e d) $500 \mathrm{MHz}$.

Os tambores preenchidos com água salgada (alvos 4, 5 e 11) são caracterizados por fortes refletores gerados no topo dos alvos, com polaridade do sinal invertida semelhante aos tambores preenchidos com água doce. Por outro lado, a base do tambor não é identificada, devido à atenuação da onda eletromagnética na água, que é muito condutiva.

0 cano metálico, implementado a 0,05 m de profundidade e na posição de $15 \mathrm{~m} \mathrm{~N}$-S, serviu como alvo-guia e foi utilizado como um referencial para todas as sete linhas instaladas no SCGR do IAG. 0 topo do cano-metálico é caracterizado por uma forte reflexão devido à elevada condutividade elétrica do metal, provocando uma reflexão total da onda eletromagnética.

As simulações numéricas geradas com as antenas de 200 e $500 \mathrm{MHz}$ (Figura 3c e 3d) mostram que com 0 uso de frequiências mais elevadas, ocorre uma diminuição da profundidade de investigação. Entretanto, há um maior detalhamento vertical dos alvos. Com o aumento das freqüências, as reverberações nos tambores com água doce e com água salgada, também aumentam devido 0 uso da fonte da onda eletromagnética do tipo exploding reflector e pelo menor comprimento de onda. 
Tabela 3 - Profundidade e tempo teórico para a reflexão das ondas eletromagnéticas no topo e na base dos tambores plásticos.

\begin{tabular}{|l|c|c|c|}
\hline \multicolumn{1}{|c|}{ Alvos } & Prof (m) Topo & T (ns) Topo & T (ns) Base \\
\hline 1 - Tambor vazio & 1,0 & 28,37 & 33,17 \\
\hline 2 - Tambor preenchido com água doce & 1,0 & 28,37 & 70,18 \\
\hline 3 - Tambor semipreenchido com água salgada & 0,5 & 14,28 & 16,58 \\
\hline 4 - Tambor preenchido com água salgada & 0,5 & 14,28 & 152,28 \\
\hline 5 - Tambor preenchido com água salgada & 1,5 & 42,86 & 180,86 \\
\hline 6 - Cano-guia metálico vazio & 0,5 & 14,28 & - \\
\hline 7 - Tambor preenchido com água doce & 1,5 & 42,86 & 84,67 \\
\hline 8 - Tambor vazio & 0,5 & 14,28 & 18,88 \\
\hline 9 - Tambor preenchido com água doce & 0,5 & 14,28 & 56,09 \\
\hline 10 - Tambor semipreenchido com água salgada & 0,5 & 14,28 & 16,58 \\
\hline 11 - Tambor preenchido com água salgada & 0,5 & 14,28 & 152,28 \\
\hline
\end{tabular}

\section{Seções de GPR}

As seções de GPR foram adquiridas em duas etapas: "antes" e "depois" dos tambores plásticos serem enterrados no SCGR-IAG. Os perfis adquiridos antes da instalação dos alvos tiveram como objetivo determinar as estruturas do background do subsolo, ou seja, sem a presença dos tambores. Para tanto, foram adquiridas seções common offset com as antenas de 100 e $200 \mathrm{MHz}$, e os resultados são correlacionados com o perfil litológico obtido através das trincheiras abertas para enterrar os alvos (Figura 4a).

Os resultados alcançados com as antenas blindadas de 100 $\mathrm{MHz}$ (Figura 4b) mostram refletores inclinados entre 1 e $2 \mathrm{~m}$ de profundidade, relacionados com a mudança de um solo argiloarenoso para um solo mais argiloso. A atenuação do sinal a partir de $2-2,5 \mathrm{~m}$ de profundidade indica a base do aterro, associada às características condutoras dos sedimentos da borda da Bacia de São Paulo. Para as antenas de $200 \mathrm{MHz}$, na Figura 4c somente os horizontes do solo mais superficiais puderam ser imageados devido à elevada atenuação no solo argiloso e 0 aumento da freqüência.

Após a instalação dos tambores plásticos no SCGR-IAG foram adquiridas seções de GPR com as antenas de 100, 200 e 500 $\mathrm{MHz}$. As interpretações foram realizadas de acordo com as profundidades verdadeiras dos tambores na subsuperfície. $\mathrm{Na} \mathrm{Fi-}$ gura 5 são apresentadas às seções de GPR obtidas com antenas não blindadas de $100 \mathrm{MHz}$, utilizando-se os modos passoa-passo e contínuo, com intervalo entre os traços de $0,2 \mathrm{~m}$ e $0,05 \mathrm{~m}$, respectivamente. No modo contínuo, o transporte das antenas transmissora e receptora foi feito através de um rebocador (Rodrigues, 2004).
Conforme observado na Figura 5b, a seção de GPR adquirida no modo contínuo apresenta melhor resolução espacial devido à diminuição do espaçamento entre os traços propiciando um maior detalhamento ao longo da linha. No entanto, a seção adquirida no modo passo-a-passo (Figura 5a) apresenta melhor relação sinal/ruído, devido o maior empilhamento dos traços, i.e., 256, 0 que pode ser comprovado através da análise da amplitude dos refletores mais profundos.

A Figura 6 mostra as seções de GPR adquiridas no modo contínuo com as antenas blindadas de 100 e $500 \mathrm{MHz}$, e com as antenas não blindadas de $200 \mathrm{MHz}$. A análise conjunta dos resultados é imprescindível para que se obtenha uma melhor interpretação qualitativa, visto que os resultados obtidos com diferentes antenas se complementam. Os tambores plásticos e 0 cano metálico guia são determinados pelos vértices dos refletores gerados no topo dos alvos, com exceção dos tambores preenchidos com água doce, caracterizados por reflexões geradas no topo e na base.

Após a conversão do tempo em profundidade e análise dos resultados, observa-se que os valores encontrados no radargrama para as profundidades do topo dos tambores correlacionados ao vértice dos refletores são próximos das profundidades reais, variando em torno de 0,01 m a 0,10 m (Tabela 4).

Os tambores vazios (alvos 1 e 8) são identificados através de fortes refletores, mas com as amplitudes diferenciadas. As razões que contribuem para a diminuição na amplitude do sinal no alvo 1, estão associadas: à perda de energia à medida que a propagação da onda vai atingindo maiores profundidades e à presença de materiais condutivos que caracterizam 0 aterro. No radargrama, o vértice destes refletores encontra-se em torno de 


\section{Distância (m)}

a)

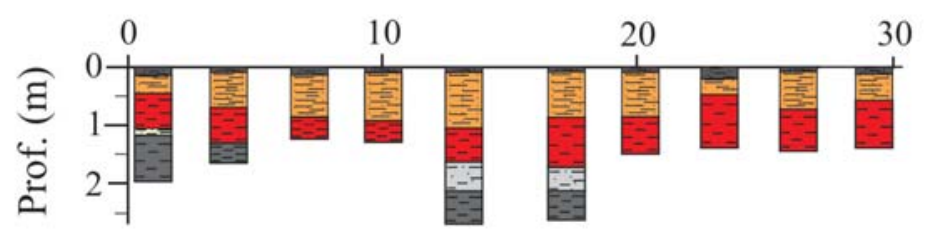

Legenda

$\begin{array}{ll} & \text { Solo areno-argiloso com matéria orgânica } \\ & \text { Solo areno-argiloso } \\ & \text { Solo argiloso-arenoso } \\ & \text { Solo argiloso } \\ E=- & \text { Solo argiloso-arenoso, fragmentos de feldspato } \\ & \text { Argila rica em matéria orgânica }\end{array}$
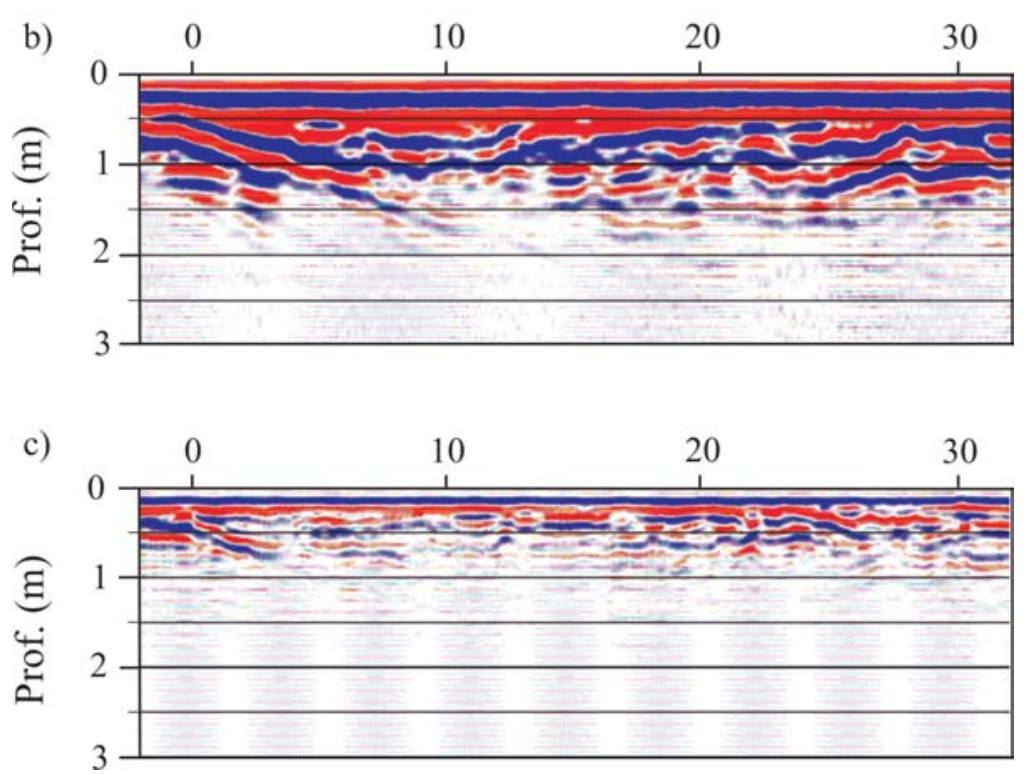

Figura 4 - a) Descrição litológica das trincheiras escavadas para a instalação dos alvos, b) Seção GPR para estabelecer o background do SCGR-IAG com as antenas de $100 \mathrm{MHz}$ e c) antenas de $200 \mathrm{MHz}$.

$1 \mathrm{~m}$ e entre 0,45 e $0,58 \mathrm{~m}$ de profundidade (Tabela 4), respectivamente. A base destes tambores não é identificada devido à velocidade de propagação da onda eletromagnética dentro do tambor (i.e., a velocidade da luz), ser maior que a velocidade no solo argiloso, ocorrendo então, uma sobreposição do $1^{\circ}$ refletor (topo) com $02^{\circ}$ refletor (base). Esse comportamento está de acordo com os resultados obtidos nas simulações numéricas (Figura 3).

Os tambores preenchidos com água doce (alvos 2, 7 e 9) foram caracterizados por refletores gerados no topo e na base dos tambores. Para 0 alvo 9, as reflexões no topo (alvo 9T) e na base (alvo 9B) são mais nítidas que nos alvos 2 e 7 que estão a maiores profundidades. A reflexão no topo apresenta menor intensidade que a da base devido à baixa impedância elétrica nesta interface e a alta atenuação do sinal, pois a condutividade elétrica do solo argiloso é maior que a da água doce. Além disso, observa-se de maneira incipiente (Figura 6a) que o refletor no topo do tambor (alvo 9T) apresenta uma polaridade invertida comparada a do topo dos tambores vazios, conforme mostrado através das simulações numéricas (Figura 3). No entanto, a base deste tambor foi caracterizada por fortes refletores seguidos de reverberações associadas à presença da água doce.

Os tambores semipreenchidos com água salgada (alvos 3T e 10T) são caracterizados por reflexões geradas no topo com intensidades similares às encontradas para os tambores vazios (alvos 

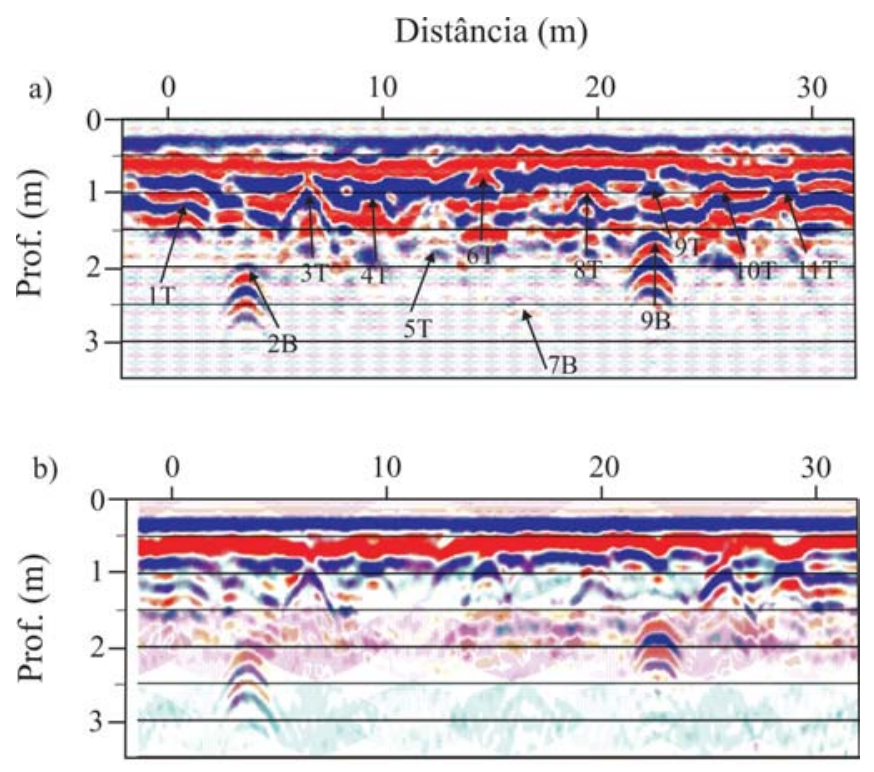

\section{Legenda}

9T - Topo do Tambor

9B - Base do Tambor

Figura 5 - Seções GPR obtidas com as antenas não blindadas de 100 MHz: a) modo passo-a-passo e b) modo contínuo.

Tabela 4 - Margem de erro entre a profundidade real dos alvos e a profundidade obtida através das seções GPR.

\begin{tabular}{|c|c|c|c|c|}
\hline Alvo & $\begin{array}{c}\text { Profundidade } \\
\text { radargrama }(\mathrm{m})\end{array}$ & $\begin{array}{c}\text { Profundidade real } \\
(\mathrm{m})\end{array}$ & $\begin{array}{c}\text { Margem de erros } \\
(\mathrm{m})\end{array}$ & $\begin{array}{c}\text { Percentual do erro } \\
\text { associado }(\%)\end{array}$ \\
\hline 1 & $0,98-1,05$ & 0,95 & $0,03-0,10$ & $3-10$ \\
\hline 2 & - & 0,99 & - & - \\
\hline 3 & $0,50-0,55$ & 0,46 & $0,04-0,09$ & $9-20$ \\
\hline 4 & $0,52-0,55$ & 0,48 & $0,04-0,07$ & $8-15$ \\
\hline 5 & $1,45-1,50$ & 1,49 & $0,01-0,04$ & $1-3$ \\
\hline 6 & $0,54-0,58$ & 0,52 & $0,02-0,06$ & $4-11$ \\
\hline 7 & - & 1,47 & - & - \\
\hline 8 & $0,45-0,58$ & 0,48 & $0,03-0,10$ & $6-21$ \\
\hline 9 & $0,48-0,58$ & 0,51 & $0,03-0,07$ & $6-14$ \\
\hline 10 & $0,48-0,58$ & 0,51 & $0,03-0,07$ & $6-14$ \\
\hline 11 & $0,48-0,55$ & 0,51 & $0,03-0,04$ & $6-8$ \\
\hline
\end{tabular}

1T e 8T). 0 topo do tambor é caracterizado pela reflexão na interface solo/plástico/ar, sendo que o limite superior da água salgada torna-se indistinguível da primeira reflexão, conforme já discutido nos resultados das simulações numéricas.

Nos tambores preenchidos com água salgada (alvos 4T, 5T e 11T) o topo dos tambores é caracterizado por fortes refletores.
Ainda que de difícil visualização, a polarização do sinal está invertida (alvo 11), de acordo com os resultados das simulações numéricas (Figura 3). 0 tambor mais profundo (alvo 5) não é identificado, devido à atenuação do sinal nos sedimentos argilosos condutivos e na água salgada dentro do tambor.

No radargrama obtido com antenas de $500 \mathrm{MHz}$ (Figura 6c) 
somente os alvos mais rasos foram caracterizados, os dispostos a profundidades maiores (alvos 2, 5 e 7) não foram identificados em razão da atenuação do sinal associada ao aumento da freqüência.

\section{Distância (m)}
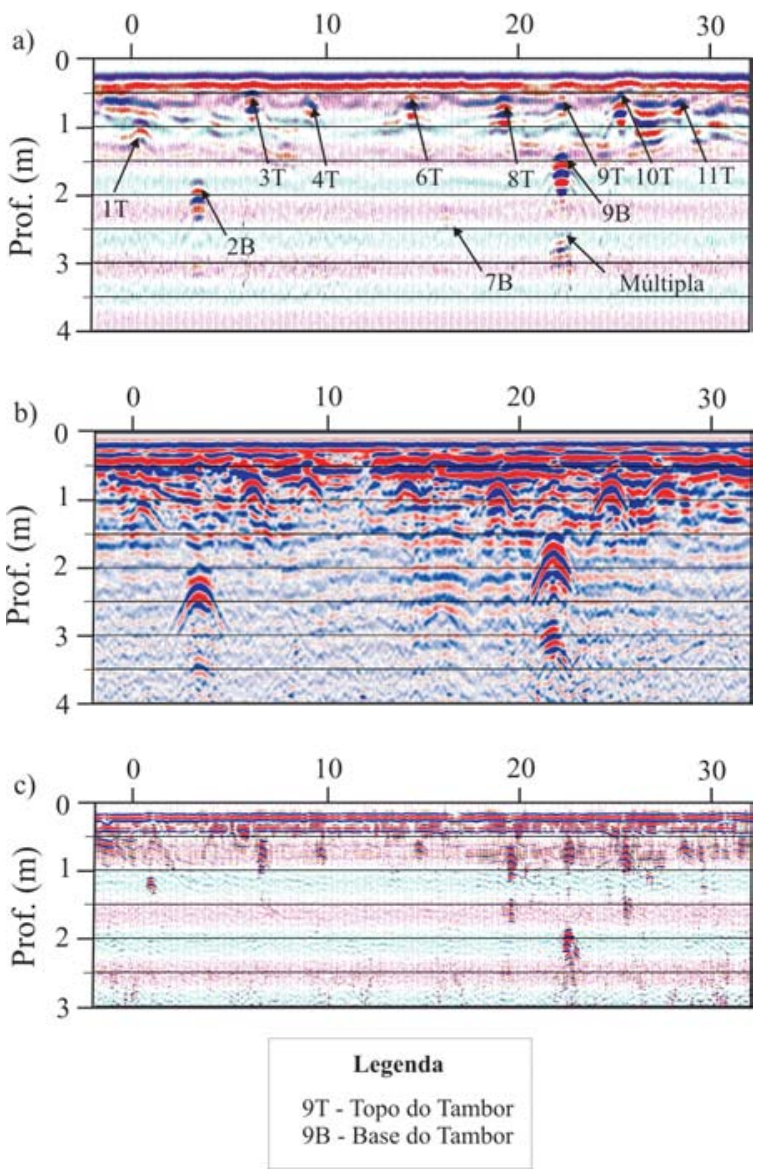

Figura 6 - Seções GPR obtidas no modo contínuo: a) antenas blindadas de 100 $\mathrm{MHz}$, b) antenas não-blindadas de $200 \mathrm{MHz}$ e c) antenas blindadas de $500 \mathrm{MHz}$.

0 cano metálico guia (alvo 6T) é caracterizado por um forte refletor hiperbólico.

Em síntese, o topo dos tambores plásticos é caracterizado por refletores que apresentam variações na intensidade do sinal, pois a reflexão está associada aos contrastes das propriedades físicas nas interfaces. De uma maneira geral, o topo dos tambores vazios, cheios de água doce, semipreenchidos com água salgada e cheios de água salgada foram caracterizados por nítidos refletores. Os tambores cheios de água doce apresentaram reflexões no topo e na base devido à menor velocidade de propagação da onda eletromagnética na água.

Tanto as antenas de altas como as de baixas freqüências são importantes na caracterização dos alvos em subsuperfície, pois os resultados se complementam. A identificação do topo dos alvos torna-se mais precisa com as antenas de altas freqüências e as de baixas freqüências permitem caracterizar os alvos mais profundos.

Quanto aos resultados obtidos com as antenas não blindadas e blindadas, vale ressaltar que embora a área de estudos localizase no ambiente urbano repleto de fontes de interferências, estas não inviabilizaram os estudos com as antenas não-blindadas. Todavia, em áreas onde há muitas interferências na superfície, recomenda-se a utilização de antenas blindadas para reduzir os efeitos das reflexões indesejadas provenientes da superfície.

\section{CONCLUSÕES}

As modelagens numéricas bidimensionais foram significativas para 0 desenvolvimento do trabalho auxiliando na minimização das incertezas a partir das assinaturas geofísicas específicas para cada tipo de material e seu conteúdo. Os resultados sintéticos apresentam uma excelente concordância com os dados reais e profundidades dos alvos. A presença de inversões de polaridade nos radargramas sintéticos foi associada ao tipo de conteúdo dos tambores.

No modo de aquisição passo-a-passo, foi obtido uma meIhor relação sinal/ruído, associada ao maior empilhamento dos traços, onde as reflexões produzidas na interface dos alvos mais profundos, apresentaram maior intensidade que a dos dados adquiridos no modo contínuo. Em contrapartida, no modo contínuo, obteve-se uma melhor resolução espacial com a diminuição do espaçamento entre os traços.

Os tambores plásticos foram bem caracterizados com as antenas de 100, 200 e $500 \mathrm{MHz}$. Com exceção dos tambores preenchidos com água doce, que foram caracterizados por reflexões geradas no topo e na base, os demais foram identificados por refletores gerados no topo dos alvos. Todas as antenas foram importantes na caracterização dos alvos. Entretanto, os melhores resultados foram obtidos com as antenas de 100 e $200 \mathrm{MHz}$.

É importante ressaltar que as comparações entre as seções de GPR antes e depois da instalação dos alvos na subsuperfície permitiriam inferir que as perturbações inseridas no subsolo foram pontuais e dessa forma, não comprometeram a disposição lateral do background da área.

Os resultados qualitativos e quantitativos sobre os alvos conhecidos, poderão ser utilizados em locais onde há pouca ou nenhuma informação sobre a subsuperfície, auxiliando estudos sobre contaminação ambiental e trabalhos voltados para a geotecnia e planejamento urbano.

Para finalizar, o uso de antenas não blindadas no contexto urbano, deve ser realizado de forma cuidadosa para que não haja 
falsas interpretações, pois nessas áreas há muitas interferências. Recomenda-se que todas as interferências na superfície sejam identificadas para posteriormente, auxiliar no processamento e análise dos resultados.

\section{AGRADECIMENTOS}

Ao Depto. de Geofísica do IAG-USP pela oportunidade de realizar 0 mestrado e a CAPES pela bolsa de pesquisa. À FAPESP pelo apoio financeiro para a instalação do SCGR do IAG-USP (Processo No. 02/07509-1). Ao Professor Francisco Hiodo, pelo desenvolvimento do rebocador de antenas não blindadas do GPR utilizado na aquisição dos dados. Ao técnico Ernande Costa Santos, à mestra Elizete Maria Araújo da Silva e ao doutorando Welitom Rodrigues Borges, pela assistência nos trabalhos de campo.

\section{REFERÊNCIAS}

ANNAN AP. 1992. Ground Penetration Radar Workshop notes. Sensors \& Software, Inc., Internal Report, $130 \mathrm{pp}$.

ATEKWANA EA, SAUCK WA \& WERKEMA DD. 2000. Investigations of geoelectrical signatures at a hydrocarbon contaminated site. Journal of Applied Geophysics, 44(2-3): 167-180.

BELÉM AN. 2001. Caracterização bidimensional de canais de rádio através de diferenças finitas no domínio do tempo. Dissertação de Mestrado, Departamento de Engenharia Eletrônica, Escola de Engenharia, Universidade Federal de Minas Gerais, Minas Gerais, 124 pp.

BORGES WR. 2002. Investigações geofísicas na borda da Bacia Sedimentar de São Paulo, utilizando-se GPR e Eletrorresistividade. Dissertação de Mestrado. Instituto de Astronomia, Geofísica e Ciências Atmostéricas, Universidade de São Paulo, São Paulo, 153 pp.

BORGES WR, PORSANI JL, SILVA EMA, ELIS VR \& HIODO FY. 2004. Utilização de Tomografia Elétrica na Identificação da Interface entre uma Cobertura Antrópica e Sedimentos Fluviais na Área do Sítio Controlado de Geofísica Rasa do IAG/USP. In: $1^{\circ}$ Simpósio Brasileiro de Geofísica, São Paulo: SBGf, CD-ROM.

BOWDERS Jr JJ, KOERNER RM \& LORD Jr AE. 1982. Buried container detection using ground-probing radar. Journal of Hazardous Materials, 7(1): 1-17.

BURGER HR. 1992. Exploration Geophysics of the shallow subsurface. Prentice Hall PTR. Englewood Ciffs, New Jersey. 489 pp.

COSTA FP. 2002. Solução numérica de equações diferenciais. Mini curso. Departamento de Ciências Exatas e Tecnológicas, Universidade Estadual de Santa Cruz, Santa Catarina, 22 pp.

DANIELS DJ. 1996. Surface penetrating radar. The Institution of Electrical Engineers, London, United Kingdom, 300 pp.
DANIELS JJ, ROBERTS R \& VENDEL M. 1994. Ground Penetration Radar for the detection of liquids contaminants. Journal of Applied Geophysics, 33: 195-207.

DAVIS JL \& ANNAN AP. 1989. Ground penetrating radar for high resolution mapping of soil and rock stratigraphy. Geophysical Prospecting, 37: 531-551.

GRANDJEAN G, GOURRY JC \& BITRI A. 2000. Evaluation of GPR techniques for civil-engineering applications: study on a test site. Journal of Applied Geophysics, 45: 141-156.

LORD Jr AE \& KOERNER RM. 1988. Nondestructive testing (NDT) techniques to detect contained subsurface hazardous waste. Journal of $\mathrm{Ha}-$ zardous Materials, 19(1): 119-123.

MABEE SB \& BROWN L. 2003. Dedicated hydrogeology/geophysical field laboratory for enhancing undergraduate education (submitted to Journal of Geoscience Education). Disponível em: $<$ <ttp://www.geo.umass.edu/dept_info/hydro/hyphy.html>. Acesso em: 21 abr. 2003

OLHOEFT GR, POWERS MH \& CAPRON DE. 1994. Buried object detection with Ground Penetrating Radar: in Proc. Of Unexploded Ordnance (UXO) detection and range remediation conference, Golden, CO, May 1719, 207-233.

PORSANI JL. 1999. Ground Penetrating Radar (GPR): Proposta metodológica de emprego em estudos geológico-geotécnicos nas regiões de Rio Claro e Descalvado -SP. Tese de Doutorado, Instituto de Geociências e Ciências Exatas, Campus de Rio Claro, Universidade Estadual Paulista - São Paulo, 145 pp.

PORSANI JL, MALAGUTTI FILHO W, ELIS VR, FISSEHA S, DOURADO JC \& MOURA HP. 2004a. The Use of GPR and VES in Delineating a Contamination Plume in a Landfill site: a Case Study in SE Brazil. Journal of Applied Geophysics, 55(3-4): 199-209.

PORSANI JL, BORGES WR, ELIS VR, DIOGO LA, HIODO FY, MARRANO A \& BIRELLI CA. 2004b. Investigações Geofísicas de Superfície e de Poço no Sítio Controlado de Geofísica rasa do IAG/USP. Revista Brasileira de Geofísica, Rio de Janeiro, 22(3): 245-258.

PORSANI JL, BORGES WR, RODRIGUES SI \& HIODO FY. 2006. 0 Sítio controlado de geofísica rasa do IAG/USP: Instalação e resultados GPR 2D-3D. Revista Brasileira de Geofísica, 24(1): 49-61.

REITZ J, MILFORD FJ \& CHRISTY RW. 1982. Fundamentos de Teoria Eletromagnética, Tradução René Balduino Sander; Carlos Duarte. Editora Campus Ltda. Rio de Janeiro, $3^{\mathrm{a}}$ edição, 516 pp.

REYNOLDS JM. 1997. Introduction to applied and environmental geophysics. Chichester: John Wiley \& Sons: 682-745.

RODRIGUES SI. 2004. Caracterização GPR de tambores metálicos e plásticos: estudo sobre o sitio controlado do IAG-USP. Dissertação de Mestrado. Instituto de Astronomia, Geofísica e Ciências Atmosféricas, Universidade de São Paulo, São Paulo. 89 pp. 
SANDMEIER KJ. 2004. ReflexW Version 3.5. Program for processing of seismic, acoustic or electromagnetic reflection, refraction and transmission data. Software Manual. Karlsruhe, Germany, 345 pp.

SAUCK W. 1996. Controlled Site for Shallow Geophysics. Institute for Water Sciences, Department of Geosciences, Western Michigan University. Disponível em:

$<$ http://www.geology.wmich.edu/fhydro/Gptest1.htm>. Acesso em: 20 abr. 2003.

SILVA EMA. 2004. Aplicação de resistividade e polarização induzida na investigação de objetos enterrados em Sítio Controlado do IAG/USP. Dissertação de Mestrado Instituto de Astronomia, Geofísica e Ciências Atmosféricas, Universidade de São Paulo, São Paulo.

SZETO AMK. 1996. York University Environmental Test Site (YES). Department of Earth and Space Science and Engineering, York University, Ontario. Disponível em: <http://www.yorku.ca/esse/yes>. Acesso em: 14 mar. 2004.

TAFLOVE A \& HAGNESS SC. 2000. Computation electrodynamics: the Finite-Difference Time Domain Method. $2^{\text {nd }}$ ed. Boston: Artech House, 852 pp.

VERSTEEG R. 1996. Optimization of GPR acquisition and noise elimination parameters. In: International Conference on Ground Penetrating Radar, 6, 1996, Sendai. Proceedings, Sendai: GPR'96, 289-292.

WARD SH \& HOHMANN GW. 1987. Electromagnetic theory for geophysical applications: Electromagnetic Methods in Applied Geophysics. Tulsa: Society of Exploration Geophysicists. In: NABIGHIAN MN (Ed.). 1(3): 131-311.

YEE KS. 1966. Numerical solution of initial boundary value problems involving Maxwell's equations in isotropic media. IEEE Trans. Antennas and Propagation, vol. Ap-4, n.3, 302-307.

YILMAZ 0. 1987. Seismic Data Processing. Tulsa: Society of Exploration Geophysicists Press, 526 pp.

ZENG X \& McMECHAN GA. 1997. GPR characterization of buried tanks and pipes. Geophysics, 62(3): 797-806.

\section{NOTAS SOBRE OS AUTORES}

Selma Isabel Rodrigues. Formada em Tecnologia Mecânica pela FATEC-SP, 2001. Mestra em Ciências, área de Geofísica Aplicada, pelo Instituto de Astronomia, Geofísica e Ciências Atmosféricas da Universidade de São Paulo - IAG/USP (2004) desenvolvendo a pesquisa "Caracterização GPR de tambores metálicos e plásticos: estudo sobre o sitio controlado do IAG-USP". Em 2004, trabalhou como Geofísica junto a Empresa IDS-Radar Ltda utilizando métodos de investigação rasa, elétricos e eletromagnéticos. Atualmente, é aluna de doutoramento do Programa de Pós-Graduação em Geofísica do IAG/USP, desenvolvendo a pesquisa "Caracterização geofísica de sítios arqueológicos de sambaquis fluviais na Região de Santa Catarina, utilizando os métodos GPR e eletromagnético indutivo".

Jorge Luís Porsani. Geólogo pelo Instituto de Geociências da UFBa (1987). Mestrado em Geofísica pelo Núcleo de Pesquisas Geofísicas Aplicadas à Prospecção de Hidrocarbonetos da UFPa (1991). De 1991 a 1996, trabalhou como Geofísico no Centro de Pesquisas da PETROBRAS. Doutorado em Geociências e Meio Ambiente pelo Instituto de Geociências e Ciências Exatas da UNESP (1999). Pós-Doutorado na Western Michigan University-USA no período de julho-novembro/2005 (Fapesp 04/13609-4). Desde 01/12/1998 é Docente do Departamento de Geofísica do IAG/USP, atuando com métodos elétricos e eletromagnéticos (GPR) aplicados à geologia, geotecnia, exploração mineral, meio ambiente e arqueologia. Coordenou o Projeto de Instalação do Sítio Controlado de Geofísica Rasa-SCGR do IAG/USP (Fapesp 02/07509-1) 